\title{
Rezension zu: Burkhard Meißner, Hellenismus (Darmstadt 2007).
}

\author{
Jörn Kobes
}

In der Reihe »Geschichte Kompakt«, die sich zum Ziel gesetzt hat, knappe, thematisch eindeutig bestimmte Aspekte der historischen Wissenschaften vorzustellen, die sich auch und vor allem an die Fachstudierenden - weniger den Fachwissenschaftler - wendet, ist dieser Abriß der hellenistischen Geschichte, mit B. MEISSNER von einem ausgewiesenen Fachmann verfaßt, erschienen. Um dieses schon jetzt festzustellen: ihm ist es dabei gelungen, ein konzises und facettenreiches Bild einer der interessantesten und umstrittensten Epochen der griechisch-römischen Antike auf kleinstem Raum und doch in kompakter Ausführlichkeit zu entwerfen. Dies ist desto deutlicher herauszuheben, wenn man bedenkt, dass auf knapp anderthalbhundert Seiten annähernd 300 Jahre historischer Ablauf im gesamten Mittelmeerraum dargestellt werden. ${ }^{1}$ Dass der vorgegebene Rahmen nun keine neuartige oder innovative Darstellungsform erwarten lassen kann, wird dem Leser von MEISSNER schon in der Einleitung verdeutlicht, wenn er sich fast entschuldigend dazu bekennt, hier die traditionelle Darstellungsform, den chronologischen Abriß, als Basis seiner Gestaltung gewählt zu haben. Natürlich hätte man auch einen systematischen Ansatz wählen können; wenn man aber berücksichtigt, dass das »Lexikon zum Hellenismus « ${ }^{2}$, das 2005 erschienen ist, gegenüber seinem direkten Vorgänger, dem $»$ Kleinen Wörterbuch des Hellenismus « ${ }^{3}$, nicht nur im Umfang, sondern auch im Format zugelegt hatte, um das Material zu erfassen, so ist die hier gezeigte Leistung nicht zu gering zu werten.

Der Hellenismus beginnt für MEISSNER mit dem Tod Alexanders des Großen ein früherer Ansatz müsste schon unter Philipp II. von Makedonien erfolgen, da bereits mit ihm neuartige Entwicklungen nicht nur in Griechenland, sondern auch in den wechselseitigen Beziehungen der Völker rund ums Mittelmeer begonnen haben. Dagegen sei der Tod Alexanders und das Fehlen eines legitimen, volljährigen und im Besitz der körperlichen und geistigen Kraft befindlichen Thronfolgers eine Hypothek für einen Neuanfang. Mit einem gesunden Selbstbewusstsein und einem Machtegoismus der Generäle des makedonischen Königs begann schließlich eine neue Zeit, von der man nicht wußte, was sie bringen sollte. Außerdem zog der Tod Alexanders Jahrzehnte lange Kriegsperioden nach sich, die nur selten von Phasen des Friedens unterbrochen wurden.

Der herkömmlichen Darstellung stellt MEISSNER eine kurze Begründung gegenüber, warum der Hellenismus für ihn erst mit dem Tod Alexanders beginnt. Es mag müßig sein, über den Beginn zu diskutieren. Das schlagende Argument, dass weder mit Alexander noch mit Philipp etwas Neues begonnen habe, sondern dass der Krieg gegen die Achämenidenherrscher hier den Ausschlag gegeben habe, greift natürlich nur, wenn man den in Aussicht gestellten Preserkrieg und die Einigung Griechenlands im Korinthischen Bund als Ausgangspunkt für den Hellenismus akzeptiert.

\footnotetext{
${ }^{1}$ Zwei Besprechungen sind mir bisher bekannt geworden: Glenn Richard Bugh: Rezension von: Burkhard Meißner: Hellenismus, Darmstadt: WBG 2007, in: sehepunkte 8 (2008), Nr. 9 [15.09.2008], URL: http://www.sehepunkte.de/2008/09/12868.html; Dorit Engster: Rezension von Burkhard Meißner, in: H-Soz-Kult vom 20.11.2007, URL: http://hsozkult.geschichte.huberlin.de/rezensionen/type $=$ rezbuecher\&id $=9936 \&$ verlage $=3$.

${ }^{2}$ Hatto H. Schmitt, Ernst Vogt (Hrsg.): Lexikon des Hellenismus. Wiesbaden 2005.

${ }^{3}$ Hatto H. Schmitt, Ernst Vogt (Hrsg.): Kleines Lexikon des Hellenismus. Wiesbaden 22003.
} 
Am Beginn des Hellenismus steht ein Legitimationsdefizit (S. 4), nämlich die offene und heiß diskutierte, später umkämpfte Frage nach der Nachfolge im Königshaus; denn als Alexander im Sterben lag, lebte kein vollwertig anerkannter Nachfolger - Philipp Arrhidaios, sein Stiefbruder, war dem Vernehmen nach geisteskrank, das Kind, das Alexander von Roxane erwartete, mußte nicht zwangsläufig ein männlicher Erbe werden. So traten die Generäle und Vertrauten Alexanders auf den Plan und konnten ihre Ansprüche, unabhängig davon, ob sie denn schon im Laufe der 2. Hälfte des Jahres 323 artikuliert wurden, erst im harten Kampf gegeneinander und in wechselnden Koalitionen durchsetzen. Dies zog sich dann in wechselvollen Koalitionen bis zur ersten richtungweisenden Entscheidungsschlacht bei Ipsos (301) und dann der Schlacht von Kurupedion (281) hin, mit der die letzten makedonischen Generäle Philipps und Alexanders hatten abtreten müssen.

Insofern wird dieses Epochendatum auch für MEISSNER zur Bruchkante, nach der sich die Entwicklung des griechisch geprägten Raumes in verschiedene Monarchien, Bünde und existentielle Blöcke aufteilte. Konnte man bis 281 noch von einem historischen Raum sprechen, so divergierten nun die Entwicklungen im ptolemäischen und selekuidischen Reich - auch wenn es noch genügend Anknüpfungspunkte wie Koalitionen, Heiratsverbindungen, territoriale Ansprüche gegen andere Monarchien geben sollte. Deswegen ändert MEISSNER nun auch in der Darstellung seinen Blick von der reinen chronologischen Erzählweise, die den gesamten Raum des Alexanderreiches im Blick hat, zu den herausgebildeten Einzelreichen am Mittelmeer, deren Abschluß er vorerst für das Jahr 217 sieht. Der Darstellung der historischen Ereignisse im antigonidischen Reich (S. 19-36) folgt ein kürzerer zum Ptolemäerreich (S. 36-45), den Schluss bilden das Seleukidenreich (S. 45-48) und die kleineren Reiche in Kleinasien sowie auf Sizilien (S. 49-51).

Der nächste Abschnitt (S. 52 ff.) ist dem Ausgreifen Roms in den griechischen Raum (220/217 v. Chr) gewidmet und führt bis zur Einrichtung der Provinz Asia nach dem Tod des letzten pergamenischen Herrschers (133). Dieser Zeitraum ist insbesondere für die politische Entwicklung der römischen Republik und mit der weiteren Entwicklung bis zum Jahr $30 \mathrm{v}$. Chr. (4. Kapitel) als Vorstufe zum römischen Prinzipat nicht zu gering zu schätzen. Eingerahmt wird dieser Abschnitt von der gegen Rom intendierten Bündnisverpflichtung des Hannibal mit dem antigonidischen König Philipp V. Dieses Bündnis und seine Folgen bescherte Rom nicht nur einen zusätzlichen Feind, der sich allerdings nicht aktiv in den Krieg in Italien einbrachte, sondern konfrontierte Rom nach dem glücklichen Ende des Krieges gegen Hannibal sehr schnell mit den Konfliktherden im östlichen Mittelmeer. Hier war mehr und mehr Roms Eingreifen gefordert, da die mit den griechischen Mächten gegen Philipp geschlossenen Freundschafts- und Bündnisabkommen die römischen Vertragspartner zur aktiven Teilnahme zwangen.

Der dritte Teil des Buches ist dann den systematisch $\mathrm{zu}$ erschließenden Aspekten dieser Epoche gewidmet. Hier untersucht MEISSNER die strukturellen Voraussetzungen hellenistischer Politik sowie ihre Entwicklungen, Adaptionen, Änderungen und Schwachstellen, die sich in jedem hellenistischen Königreich anders darstellen konnten. Ein standardisiertes „hellenistisches Königreich“ war ebenso unmöglich wie schon die Tatsache, nicht voraussehen zu können, wie sich die Staaten entwickeln sollten. Jeder Herrscher - selbst wenn wir die kleineren Dynastien in Kleinasien berücksichtigen - mußte neben den grundsätzlichen Machtverhältnissen immer wieder auch die geographischen, kulturellen und personalen Bedingungen in seine Überlegungen einbeziehen. 
In fünf weiteren Abschnitten legt MEISSNER, nun losgelöst vom chronologischen Gerüst, die Entwicklungen dar in Bezug auf das Königtum, Recht und Verwaltung, Wirtschaft und Gesellschaft, Wissenschaft und Kultur sowie Religion und typisch hellenistischen Erscheinungen. Dabei betont MEISSNER das charakteristische Kennzeichen des Hellenismus: die Kombination althergebrachter Institutionen mit neuen Formen und Inhalten, hinter der sich jedoch unter der Hülle des Bewährten eine Menge an Veränderungen sowie Mutationen, aber auch radikale Neuerscheinungen verbergen. Zwar existierte der „Bürgerverbandsstaat“ in Form der Polis weiter; daneben jedoch gewannen die Königreiche, die Verbünde der vordergründig selbstständigen Städte und die Stammesverbände die Entscheidungsgewalten - die Poleis wurden teils freiwillig, teils unter Druck oder durch militärische Gewalt in die jeweilige staatliche Organisation integriert. So gewannen vor allem die von imposanten Gründungsaktivitäten begleiteten hellenistischen Neugründungen Alexanders, die seine Nachfolger gelegentlich zu übertreffen versuchten, eine Position, die die alt-ehrwürdigen Städte im griechischen Kernland hergeben mussten. Die Entscheidungszentren verschoben sich von Griechenland nach Makedonien, nach Syrien, nach Asien und nach Ägypten - die ehemals einflussreichen griechischen Städte wurden zu Spielbällen der Monarchien, auch wenn sie im weiteren Verlauf den Eindruck erwecken konnten, im „Vollbesitz ihrer territorialen Gewalten“" zu sein.

Das hellenistische Königtum kann mit MEISSNER durch zwei Begriffe treffend charkterisiert werden: die „,charismatische Herrschaft“ (nach H.-J. Gehrke) und das „speergewonnene Land“ (nach A. Mehl). Beide Begriffe, die aufgrund der vordergründig militärischen Legitimation der Heerführer Alexanders in dessen Nachfolge gegenüber der dynastischen Erbfolge propagiert werden, umschreiben die Kernkompetenz der hellenistischen Könige, zumindest in den beiden ersten Generationen (mit Abstrichen) nach Alexanders Tod: Militärische Erfolge - und seien sie noch so zweifelhaft oder nur kurzfristig errungen - und die damit verbundene Anerkennung als „Anführer“ durch die sie legitimierenden Heere brachte ihnen das Diadem ein, das sie verteidigen und ihren Nachkommen - hier wird das Charisma des siegreichen Herrschers durch die familiäre Nachfolgeregelung abgelöst - hinterlassen konnten.

Im nächsten Abschnitt des 3. Teils geht MEISSNER kurz auf den Aspekt „Recht und Verwaltung" ein; hier stellt er summarisch den ptolemäischen Verwaltungsapparat und die Stellung der einheimischen Bevölkerung im Gegensatz zu den griechischen Bewohnern sowie die Sonderstellung Alexandrias dar. Ebenso schematisch, aber mit einer entsprechenden epigraphischen Quelle (Brief des Antigonos Monophthalmos an die kleinasiatische Stadt Skepsis) wird das griechische Kerngebiet abgehandelt; nur die entsprechenden Hinweise auf das seleukidische Reich, das sich neben der makedonischen Verwaltung auch auf achämenidische Wurzeln berufen konnte, sind der knappen Darstellung zum Opfer gefallen. Gleichermaßen kurz werden anschließend „Wirtschaft und Gesellschaft" beleuchtet hier liegt der Schwerpunkt wegen der vorteilhaften Quellenlage auf Ägypten; daneben finden sich einige Bemerkungen zum Seleukidenreich.

Ausführlicher geht MEISSNER dann im nächsten Kapitel (III 4.) auf „Wissenschaft, Technik, Kultur und Literatur“ ein - allesamt Schwerpunktinteressen des Autors. Dabei gelingt es M. wiederum sehr gut, auf engstem Raum die Entwicklungen in hellenistischer Geschichtsbeschreibung und Dichtung, Philosophie, Wissenschaft, Weiterentwicklung von Technik und Technologie-Transfer darzustellen. MEISSNER kann hier anschaulich zeigen, dass aufgrund des 
Alexanderzuges viel griechisches Wissen nach Osten, ebenso aber auch nichtgriechisches Wissen nach Westen gelangte, dort adaptiert, verfeinert und weiterentwickelt wurde, um dann wieder im Rahmen von Transferleistungen in die ursprünglichen Herkunftsländer zurück zu gelangen. Exemplarisch lässt dies sich im Bereich „Technik und Technologie“ auf dem zivilen Sektor „Wasser- und Landwirtschaft" sowie im militärischen Bereich feststellen. Ohne die beeindruckende Kriegsmacht, die mitgeführten Werkzeuge, Tiere und das notwendige Know-how, das man erweiterte, wenn die Situation es erforderlich machte, wären einige militärische Aktionen (Belagerung von Tyros, Alexanderzug, Erfindungen des Archimedes) im Hellenismus nicht möglich gewesen.

Das letzte Kapitel widmet sich dem Thema „Religion und Lebensformen“. Hier nimmt der hellenistische Herrscherkult einen zentralen Platz ein. Von Alexanders Siwabesuch (332 v. Chr.) ausgehend werden knapp die religiösen Implikationen besprochen, denen die ptolemäischen Herrscher im Einklang mit und im Kontrast zu den altägyptischen Göttervorstellungen ausgesetzt waren. Dabei nimmt das sog. Kanopus-Dekret eine herausgehobene Stellung ein, um das Zusammenwirken zwischen alter und neuer Religion, altem und neuem Herrscherkult sowie die Einbindung der königlichen Familie in einen ägyptischen Kult anhand des ägyptischen Kultkalenders darzustellen. ${ }^{4}$

Auch andere Entwicklungen im Herrscherkult finden Erwähnung, so Demetrios Poliorketes' Einzug in Athen, der der Bevölkerung als Ankunft eines lebenden Gottes „,verkauft“ wurde, um in der damals für Athen bedrohlichen Phase das Eingreifen des Königs noch weiter zu überhöhen, ihn göttergleich zu machen; denn er sei „,der einzige wirkliche Gott“ (Athen. Deip. VI 63 p. 253 d-f nach Duris von Samos, FGrHist 76 F 13), da nur er, aber nicht die anderen Götter, die Stadt aufsuchte und ihr half. Eine andere Ausformung des Herrscherkultes, die MEISSNER nicht unerwähnt läßt, ist die späthellenistische Götterterasse der Könige von Kommagene am Nemrud Dağ. Spätestens hiermit war es der Herrscherfamilie gelungen, griechisches Gedankengut mit iranischen Göttervorstellungen zu einem Ensemble zu verbinden, das auch sinnstiftend für die untergebene Bevölkerung werden konnte. Es gelang ihnen, diesen Synkretismus als ,ein Instrument zur Relativierung kultureller und religiöser Differenzen“" (S. 129) zu schaffen.

Dass die Kombination griechischer und einheimischer Kultvorstellungen jedoch nicht immer reibungslos gelang, beweisen hinlänglich die Auseinandersetzungen zwischen den Seleukiden und den Hohepriestern in Jerusalem in den sog. Makkabäeraufständen, die schließlich den Herrschern Grenzen aufzeigten und der Bevölkerung Möglichkeiten eröffneten, ein gewisses Maß an Selbstständigkeit zu gewinnen. Dass damit jedoch keine Beruhigung der politischen Situation einhergehen musste, muss nicht eigens betont werden. Während ein griechisch erzogenes Volk mit einer Gleichsetzung des Herrschers mit einem Gott keinerlei gravierende Probleme haben musste und deswegen der Kultausübung an exponierten Plätzen der Polis zustimmte, war es in den Augen der Angehörigen einer monotheistischen Religion, wie es das Judentum darstellt, reine Blasphemie und forderte deshalb zum Widerstand heraus, der nebenbei die personalen Interessen großzügig verdecken konnte (Makkabäerbücher; Daniel-Buch).

Zwei weitere Begriffe, die MEISSNER zum Abschluß seiner Überblicksdarstellung aufgreift, verweisen wiederum auf das Selbstverständnis des

\footnotetext{
${ }^{4}$ Hier ist als wichtiger bibliographischer Nachweis nachzutragen: Stefan Pfeiffer, Das Dekret von Kanopus (238 v. Chr.). Kommentar und historische Auswertung (Archiv für Papyrusforschung, Beiheft 18), München/Leipzig 2004.
} 
„Hellenismus“, nämlich der Kosmopolitismus des einzelnen Bürgers, der neben seiner Zugehörigkeit zur Polis (wie eh und je) natürlich auch Reichsbewohner und griechisch sprechender Einwohner gewesen ist, sowie das Gymnasion. Dieses Phänomen wurde durch den Alexanderzug als ein wichtiges Kennzeichen einer Stadt griechischer Ausprägung bestimmend und war selbst in den entlegendsten Gegenden der Herrscherbereiche in den Grenzen des früheren Alexanderreiches zu finden, wo das Gymnasion als Katalysator griechischer Erziehung wahrscheinlich weit mehr Einfluss erlangen konnte als eine militärische Intervention. ${ }^{5}$

Als Kritikpunkt ist anzumerken, dass der vom Verlag konzipierte Klappentext zwar wie für die anderen Bände der Reihe auch ein kommentiertes Quellen- und Literaturverzeichnis verspricht, das man in diesem Buch jedoch vergeblich sucht. Stattdessen findet der Leser eine 10-seitige Auswahlbibliographie. Ferner sind dem Rezensenten lediglich kleinere Fehler hinsichtlich Orthographie (z. B. S. 11: Magara statt Megara; S. 29 die Zitierweise der Inscriptiones Graecae zum ChremonidesAntrag in Athen) und Sortierung (z. B. S. 139: Literaturverzeichnis unter dem Topos »Diadochen und Epigonen«) sowie Inkonsequenzen bei den verwendeten Namen moderner Autoren ${ }^{6}$ aufgefallen. Auch bei Wortrennungen hätte durch ein sorgfältiges Lektorat das eine oder andere vermieden werden können (s. S. 28: Ale-xander; S. 29: IG II/III2 statt IG II/III').

Aber dies sind - zugegeben - nur Marginalien. Der Wert der Studie für den akademischen Unterricht ist nicht bestreitbar - das studentische Publikum wird gerne zu dieser Darstellung greifen, auch wenn die komprimierte Form nicht auf Anhieb alles beantworten wird.

\footnotetext{
5 Nachzutragen ist hier: Daniel Kah/Peter Scholz (Hrsg.), Das hellenistische Gymnasion. (Wissenskultur und gesellschaftlich Wandel. Bd. 8), Berlin 2004.

${ }^{6}$ Beispielsweise wird der Personenname Elias J. Bickermann mal deutsch, mal französisch, mal englisch zitiert, ohne den Leser über die Umstände, sei es in einer kurzen Fußnote, zu informieren, die zu einer solchen Namenvielfalt geführt hat.
} 